lieren, erstens an ihrer in Teilen der Partei hinterfragten Eignung für diese Position, zweitens an der innerorganisatorischen Top-Down-Einmischung in das Verfahren der Vornominierung und damit verbunden drittens daran, dass man sich über die in einem peniblen Bezirksproporz institutionalisierten landsmannschaftlichen Antagonismen hinwegzusetzen versuchte.

Martin Sebaldt führt wichtige Erkenntnisse im Schlusskapitel zusammen (S. 559 - 580). Er bescheinigt der CSU eine positive Leistungsbilanz, vor allem bei der Profilierung Bayerns als wettbewerbsfähiger Dienstleistungsstandort. Die komplexer gewordenen Beziehungen der Partei zum pluralisierten Feld intermediärer Vorfeldorganisationen kennzeichnet er als „entideologisierten Pragmatismus“ (S. 565). Gesellschaftlich ist die Partei vergleichsweise gut verankert, bei einer nachhaltigen Affinität christlich gebundener Bürger. Unverkennbar ist jedoch auch, dass die CSU auf die gelbe Karte der bayerischen Wähler zur Landtagswahl 2008 offenbar nicht ausreichend durch organisatorische Reformen und programmatische Anpassungen reagiert hat. Dennoch beziehungsweise auf Grundlage der im Band skizzierten Stärken der Organisation (insbesondere „umfassende, flächendeckende Organisation “ oder „durchorganisierter, ebenenübergreifender Aufbau“, S. 576) wird die in der Wissenschaft anerkannte "Selbstregenerationsfähigkeit“ der CSU (Andreas Kießling) nicht in Frage gestellt. Sie wird abschließend als „erfolgreiche Volkspartei“ (S. 575) eingeordnet, die für die Zukunft "gut" gerüstet sei (S. 575 - 577). Festhalten lässt sich, dass der Sammelband gehaltvolle Einblicke - längst nicht nur für Parteienforscher - in die anpassungsbewährte Organisationswirklichkeit der Ausnahmepartei CSU ermöglicht und damit die traditionsreiche CSUForschung fortschreibt.

Benjamin Höhne

\title{
Piratenpartei: rasanter Aufstieg, schnelle Analyse
}

Appelius, Stefan und Armin Fuhrer: Das Betriebssystem erneuern. Alles über die Piratenpartei, Berlin Story Verlag, Berlin 2012, 336 Seiten, € 19,80.

Häusler, Martin: Die Piratenpartei: Freiheit, die wir meinen. Neue Gesichter für die Politik, Scorpio Verlag, Berlin / München 2011, 176 Seiten, € 6,95.

Neumann, Tobias: Die Piratenpartei Deutschland. Entwicklung und Selbstverständnis, Contumax-Verlag, Berlin 2011, 272 Seiten, € 19,90 (als Paperback oder kostenfrei als E-Book).

Wer dachte, die Politikwissenschaft würde sich zu wenig den aktuellen Themen widmen, sieht sich mit der Forschung zur Piratenpartei widerlegt. Weniger als ein Jahr nach dem spektakulären Wahlerfolg der Piraten bei der Abgeordnetenhauswahl in Berlin steht schon Literatur in bemerkenswertem Umfang zur Verfügung. Wenig überraschend thematisieren bisher die meisten Werke zunächst die Entstehung der Partei.

Noch vor dem politischen Durchbruch in Berlin erschien Tobias Neumanns „Die Piratenpartei Deutschland: Entwicklung und Selbstverständnis“. Wie für eine Qualifikationsarbeit üblich - es handelt sich um eine Magisterarbeit an der Johann Wolfgang Goethe-Universität Frankfurt am Main -, hält sich der Lesegenuss in Grenzen. Interessant an dieser Arbeit sind jedoch die detaillierte Beschreibung der Genese der Partei und vor allem das darin ausgewer- 
tete Datenmaterial. Neumann - selbst Mitglied der Piratenpartei - führte eine Umfrage unter allen Parteimitgliedern mit einer respektablen Rücklaufquote durch, und zwar noch vor der Berliner Abgeordnetenhauswahl, so dass Aussagen über die Beitrittsmotive jener Mitglieder möglich sind, die der Partei mehrheitlich schon 2009 beigetreten waren.

Interessant ist zum Beispiel, dass schon zu diesem Zeitpunkt das wichtigste Motiv für einen Beitritt die Unzufriedenheit mit den etablierten Parteien war. So wurde gemäß Neumann die Debatte um die Vorratsdatenspeicherung, die allgemein als für diese erste Beitrittswelle entscheidend betrachtet wird, nur von etwa zwei Dritteln als Motiv genannt. Ebenfalls zu Recht betont Neumann, dass viele Mitglieder der bis dahin noch sehr jungen Partei politisch inaktiv waren. Neben diesen und einigen weiteren interessanten Beobachtungen erfährt man jedoch wenig über die Parteimitglieder. Insbesondere lernt man kaum etwas über deren politische Einstellungen und Werte. Zu problematisch sind viele Messinstrumente und zu sehr fokussiert der Text auf Organisation und Kommunikation innerhalb der Partei.

Martin Häuslers Band widmet sich in erster Linie der Entstehungsgeschichte der deutschen Piraten. In flottem Erzählton beschreibt er deren Ursprünge im Widerstand gegen die Durchsetzung von strengen Copyright-Regeln und staatlicher Regulierung im Internet sowie ihre Organisation als politische Partei. Ebenso wirft Häusler einen Blick auf das Programm der Piraten, ihren ersten großen Wahlerfolg in Berlin und die erste Reaktion der etablierten Parteien. Für eine breite Öffentlichkeit geschrieben, berichtet das doch recht dünne Buch - 50 der gut 170 Seiten bestehen aus dem Grundsatzprogramm der Piratenpartei - allerdings wenig Unbekanntes und dient Politikwissenschaftlern damit allenfalls als Einstiegslektüre.

Das meiste, was Häusler zu entnehmen ist, kann auch bei Stefan Appelius und Armin Fuhrer nachgelesen werden. Doch, wie schon der Titel des Bandes besagt, befindet sich in diesem Text, der ebenfalls an ein relativ breites Publikum adressiert ist, noch mehr. So findet sich eine bunte Mischung aus Faktensammlung, Erfahrungsberichten, Analyse und Porträts, deren Stärke es ist, ein recht umfangreiches Bild über die bisherige Geschichte der Piratenpartei zu zeichnen.

Was die Entstehungsgeschichte und die Programmatik betrifft, sind sich alle Autoren im Großen und Ganzen einig. Zum Beispiel gibt es keinen Zweifel über die Präferenz der Piratenpartei für mehr partizipative Demokratie. Ebenfalls sehen die verschiedenen Autoren die Partei, natürlich im Zusammenhang mit deren Haltung zur Überwachung des Internets, als gesellschaftsliberal. In allen drei Büchern werden die Piraten mit den Grünen verglichen, und es wird auf viele Übereinstimmungen und wenige Unterschiede hingewiesen.

Weniger als ein Jahr nach dem Wahlerfolg in Berlin verfügen wir also bereits über eine relativ breit gefächerte Literatur zur Piratenpartei. Doch beantwortet diese auch die für die Politikwissenschaft bedeutenden Fragen nach den Ursachen für den Wahlerfolg und der demokratietheoretischen Bedeutung des Liquid Democracy-Konzepts? Insgesamt werden diese entscheidenden Fragen in allen drei Veröffentlichungen kaum in den Vordergrund gestellt, so dass die Antworten teilweise unbefriedigend ausfallen. Häusler bringt zum Beispiel den Wahlerfolg unter anderem mit der „Verlustangst der Deutschen“ (S. 99) in Verbindung, ohne jegliche Evidenz für seine These zu präsentieren oder den kausalen Zusammenhang zwischen Verlustangst und Wählerverhalten zu präzisieren. Da scheint seine zweite Hypothese, dass die Piratenpartei vor allem wegen ihrer Forderung nach mehr direkter Demokratie erfolgreich ist, eher plausibel (S. 112, S. 118 f.) - eine Hypothese, die auch bei Appelius und Fuhrer (S. 59, S. 141 ff.) zu finden ist. Bei Letzteren wird neben der Forderung nach direkter Demokratie auch die Netzpolitik der Piratenpartei als für den Erfolg ausschlaggebend betrachtet. 
Sind die Piraten also eine Partei der internetaffinen Postmaterialisten? So plausibel diese These klingt, die erklären könnte, weshalb die Piratenpartei über ihr eigentliches Milieu hinaus mobilisieren kann: Die Daten aus den Wählerbefragungen sprechen zumindest teilweise eine andere Sprache. Denn wie geht die Hypothese, dass die Piratenpartei in erster Linie die internetaffinen Postmaterialisten mobilisiere, mit dem Umstand zusammen, dass dieselbe Partei bei den Wahlen nach Berlin einen überdurchschnittlich hohen Anteil an Arbeitslosen unter ihren Wählern aufweist? Hier braucht es doch einiges an Phantasie, um den Erfolg der Piratenpartei nicht (auch) in Verbindung mit dem Protestmotiv zu bringen. Entsprechend abenteuerlich ist Appelius' und Fuhrers Behauptung, dass „die Kategorie der Arbeitslosen bei der Piraten-Anhängerschaft nicht in erster Linie aus den klassischen HartzIV-Empfängern rekrutiert, sondern eher aus einer Gruppe, die gut ausgebildet ist, aber (noch) keine gesicherten Arbeitsverhältnisse hat" (S. 64).

Bleibt noch die Frage zum Potential von Liquid Democracy: Kann dieses Konzept Umsetzungsprobleme der direkten oder sogar deliberativen Demokratie lösen? Appelius und Fuhrer sind optimistisch. Sie gehen zurück auf die Urdemokratie Athens und versuchen zu zeigen, dass eine ähnliche Vorstellung von Demokratie, wie sie der Idee der „flüssigen Demokratie“ innewohnt, schon einmal erfolgreich umgesetzt wurde. Sie nennen jedoch zwei Probleme der direkten Demokratie Athens: Erstens, dass diese, wie auch Rousseau zu bedenken gab, nur in einer relativ kleinen Gemeinschaft umsetzbar sei. Dafür böten „Tools“ im Geiste von Liquid Democracy wie LiquidFeedback eine Lösung, indem Kommunikations- und Abstimmungsprozesse in einen virtuellen Raum verlagert werden. Zweitens drohte der direkten Demokratie athenischen Typs die Machtkonzentration bei Demagogen. Hierfür, so geben Appelius und Fuhrer zu, hätte auch das Konzept der Liquid Democracy noch keine Lösung bereit.

Insgesamt ist es der Politikwissenschaft mit Bezug auf die Piratenpartei gelungen, sehr schnell einige interessante Antworten auf ein neues Phänomen zu geben. Weiterführende Forschung in diese Richtung ist jedoch erwünscht. Insbesondere fehlt es nach wie vor an einer fundierten wahlsoziologischen Analyse, die eine überzeugende Antwort auf die Dynamik des Wahlerfolgs der Piratenpartei gibt. Auch was den demokratietheoretischen Wert des Liquid Democracy-Konzepts betrifft, bleiben noch viele Fragen offen. So wäre es interessant, dieses nicht nur im Lichte von Idealtypen partizipativer Demokratie zu betrachten, sondern mit Bezug auf die real existierenden (semi-)direkten Demokratien zu reflektieren.

Oliver Strijbis

\section{Piratenpartei: weitere Erschließung eines politischen Phänomens}

Bieber, Christoph und Claus Leggewie (Hrsg.): Unter Piraten. Erkundungen in einer neuen politischen Arena, transcript, Bielefeld 2012, 248 Seiten, € 19,80.

Niedermayer, Oskar (Hrsg.): Die Piratenpartei, VS Verlag für Sozialwissenschaften, Wiesbaden 2012, 200 Seiten, $€ 24,95$.

Berlin am 18. September 2011, 18 Uhr: Die erste Hochrechnung zur Berliner Abgeordnetenhauswahl geht von 8,9 Prozent der Zweitstimmen für die Piratenpartei aus. Dies ist das singuläre Ereignis, das eine intensive wissenschaftliche Beschäftigung mit dem jungen Phä- 